\section{Análisis de los principales conflictos éticos entre la Declaración de Helsinki 2008 y su propuesta de cambio}

\author{
SOFÍA P. SALAS ${ }^{1,2}$, MOISÉS RUSSO N. ${ }^{2}$
}

\section{Analysis of the main ethical conflicts in the 2008 declaration of Helsinki and the proposed changes in the new version}

The Declaration of Helsinki (DoH) of the World Medical Association is the basis of the rules governing research on human beings. The latest version (enacted in Korea, 2008) has been a source of ethical discussions, particularly regarding the use of placebos, the measures to assure the access of study subjects to interventions identified as beneficial once the study has ended, and the need of a better protection of potentially vulnerable groups. These issues led to draft a revised version, that was approved in 2013. In this paper, we present an ethical analysis of the main changes contained in the DoH, including the proposed changes in the new version, referring, when appropriate, to relevant Chilean laws governing research on human subjects. In our opinion, the $2008 \mathrm{DoH}$ contains significant imprecisions on some of these issues, making a new version of the declaration necessary to fully protect subjects participating in biomedical research, as stated by the new version approved in 2013.

(Rev Med Chile 2014; 142: 475-480)

Key words: Ethics, research; Helsinki declaration; Placebos; Research subjects.

\author{
'Programa de Ética y Políticas \\ Públicas en Reproducción \\ Humana. \\ ${ }^{2}$ Escuela de Medicina, Universidad \\ Diego Portales, Santiago, Chile. \\ Este trabajo no tuvo apoyo \\ financiero. \\ Nota: Parte de este artículo fue \\ presentado como trabajo inédito \\ al Premio de Ética 2012 del \\ Colegio Médico, donde obtuvo \\ Mención Honrosa. \\ Recibido el 27 de junio de 2013, \\ aceptado el 18 de octubre de \\ 2013. \\ Correspondencia a: \\ Dra. Sofía P. Salas \\ Ejército $260,2^{\circ}$ Piso; Santiago, \\ Chile. \\ Teléfono: (562) 26762829 \\ sofia.salas@udp.cl
}

L a Asociación Médica Mundial (AMM) dio a conocer la Declaración de Helsinki (DdeH) en el año 1964, la cual establece principios éticos para las investigaciones en seres humanos, incluyendo la investigación en material humano y de información identificable ${ }^{1}$. Aunque la DdeH no es vinculante, es la base de las normas que regulan la investigación en seres humanos, siendo usada extensamente por agencias regulatorias y comités de ética de la investigación en distintos países $^{2,3}$. La DdH ha tenido seis modificaciones hasta octubre de 2008, cuando la 59a Asamblea Mundial de la AMM, reunida en Corea, adoptó una nueva versión, que reemplaza a todas las anteriores ${ }^{4}$. Esta versión contiene modificaciones que han sido objeto de discusión por el impacto que pueden tener sobre la investigación clínica. Mientras algunos la consideran como una decla- ración "paternalista"5,6, la Confederación Médica Latinoamericana y del Caribe (CONFEMEL) la critica por no resguardar en forma adecuada la salud de los participantes, especialmente aquellos de países en vías de desarrollo ${ }^{7}$. Producto de esta controversia, se formó un grupo de trabajo que recientemente ha propuesto una nueva versión, la cual estuvo abierta a consulta pública entre el 15 de abril y el 15 de junio de $2013^{8}$.

La controversia sobre la DdeH 2008 recoge algunos de los aspectos esenciales del debate surgido a partir de DdeH 2000 y las subsecuentes aclaraciones a los anteriores párrafos 29 y 30 . Algunas de las modificaciones más relevantes se refieren al uso de placebo, a las medidas que se deben otorgar a los participantes al término de la investigación y a la necesidad de asegurar mayor protección a los participantes, especialmente a los grupos 
vulnerables. En el presente artículo, discutiremos los alcances bioéticos de estas modificaciones, comparando los aspectos diferenciadores más importantes entre la Declaración del 2008 y la nueva propuesta, además de destacar algunos aspectos relevantes para la investigación biomédica en Chile contenidos en la Ley 20.584 que "Regula los derechos y deberes que tienen las personas en relación con acciones vinculadas a su atención en salud" promulgada el año $2012^{9}$. Esta Ley regula varios de los aspectos que la Declaración de Helsinki se propone normar, incluyendo aquellos relacionados con investigación en personas con discapacidad psíquica o mental. Para facilitar la comprensión de estos cambios, hemos mantenido la numeración de los párrafos de la DdH 2008 y, de ser pertinente, señalamos qué cambios se han introducido en la nueva propuesta.

\section{Principales cambios en Helsinki 2008 y en propuesta actual}

\section{Consideraciones respecto del uso de placebo}

La DdH 2008 contempla restricciones al uso de placebo (Párrafo 32): "Los posibles beneficios, riesgos, costos y eficacia de toda intervención nueva deben ser evaluados mediante su comparación con la mejor intervención probada existente, excepto en las siguientes circunstancias:

- El uso de un placebo, o ningún tratamiento, es aceptable en estudios para los que no existe intervención probada existente; $o$

- Cuando por razones metodológicas, científicas $y$ apremiantes, el uso de un placebo es necesario para determinar la eficacia y la seguridad de una intervención que no implique un riesgo, efectos adversos graves o daños irreversibles para los pacientes que reciben el placebo o ningún tratamiento. Se deber tener mucho cuidado para no abusar de esta opción".

La DdH 2008 permite que bajo ciertas circunstancias un nuevo tratamiento sea probado contra placebo, aun cuando estuviese disponible una terapia eficaz. Este aspecto ha recibido fuertes críticas, puesto que se considera que el uso de placebo pone en riesgo la salud de las personas ${ }^{10}$, y no se aclara cuáles serían esas poderosas razones que justifiquen su uso, además de considerar que es redundante señalar que las razones deben ser "científicamente sólidas", puesto que toda investigación requiere de solidez científica ${ }^{11}$. En la propuesta actual, este párrafo (que pasa a ser el P33), sólo tiene cambios menores y enfatiza que los pacientes que reciban cualquier intervención menos efectiva que la mejor terapia probada no estarán expuestos a riesgo serio o irreversible como resultado de esto, especificando que existen restricciones a cualquier investigación cuyo comparador no sea el estándar aceptado.

El cuestionamiento al uso de placebo en la investigación biomédica se basa en el principio moral que ninguna persona puede ser denegada del mejor tratamiento disponible, y en la presunción de que la ética de la investigación biomédica no disminuye las responsabilidades hacia el paciente. En nuestro parecer, creemos adecuado que las restricciones al uso de placebo se mantengan, aunque implique que podría ser necesario reclutar mayor número de pacientes para probar que una nueva terapia es más efectiva que el procedimiento estándar. Debemos reconocer que no existe consenso respecto de esta postura. Por ejemplo, Carpenter considera que existe un valor superior de permitir que se identifique, para la población afectada con una determinada condición, las formas más efectivas y menos dañinas de tratamiento y que, para lograr estos objetivos, el placebo cumpliría un papel relevante ${ }^{12}$. Entre sus argumentos está el que exista una posibilidad razonable que el tratamiento experimental tendrá mayor efectividad o menor efecto adverso comparado con el tratamiento en uso y que la condición en estudio sea susceptible de fluctuaciones relevantes en su severidad. Creemos que si existe terapia considerada como efectiva, un diseño adecuado del estudio debiese permitir controlar esta variabilidad, debiéndose poder demostrar mayor efectividad de la nueva intervención, si la hubiese. Con todo, existirán situaciones en las cuales se hace necesario el uso de placebo, las cuales quedan contempladas, de manera general, en la DdH 2008.

Un aspecto controversial es si "la mejor intervención probada existente” es aquella que está disponible a nivel local o si se debiesen usar estándares internacionales para determinar cuál es el mejor comparador para una determinada condición. Al respecto, compartimos la idea general de definir al cuidado estándar como aquel cuidado que recibiría dicha población si las políticas sanitarias funcionaran correctamente en el lugar (para revisión ver referencia) ${ }^{13}$. En esta 
línea, se ha propuesto que si la investigación es financiada por agentes externos (por ejemplo, investigación multicéntrica internacional), se exijan los mismos estándares de cuidado que recibirían los participantes del estudio si éste se realiza en el país patrocinante ${ }^{13}$, mientras que si la investigación obedece a necesidades locales, el estándar de cuidado debiese estar en consonancia con la realidad del país huésped. Una política de este tipo puede permitir la utilización de un comparador por debajo de los estándares internacionales, siempre que haya real posibilidad de implementación de esa intervención en la realidad local y que la vulnerabilidad de los sujetos de investigación no esté siendo explotada para beneficios de otras poblaciones, sino que los resultados del estudio puedan ser utilizados por la misma población que ha participado en éste.

\section{Consideraciones respecto de poblaciones vulnerables}

La palabra vulnerable viene del latín vulnerabilis, formada de vulnus (herida) y el sufijo abilis que indica posibilidad ${ }^{14}$. De ahí que vulnerable significa "que puede ser herido". La consideración a la vulnerabilidad de los participantes en una investigación se relaciona con el principio de justicia respecto de la selección de participantes, las limitaciones a la capacidad de otorgar un consentimiento informado (CI) válido, y la presencia de relaciones asimétricas entre los grupos vulnerables y los investigadores y patrocinantes. Entre las múltiples poblaciones que son susceptibles de ser consideradas como vulnerables se encuentran los ancianos, personas pobres, estudiantes, prisioneros, discapacitados mentales, minorías, niños y mujeres en edad fértil, entre otros. En la DdH se optó por no especificar qué grupos son considerados como vulnerables, aunque se hace mención general a aquellos sujetos con discapacidad física o mental y a las comunidades con desventajas como merecedores de protección especial. Al respecto, la DdH 2008, en la $2^{\circ}$ parte del Párrafo 9 señala: " $A l$ gunas poblaciones sometidas a la investigación son particularmente vulnerables y necesitan protección especial. Estas incluyen a los que no pueden otorgar o rechazar el consentimiento por sí mismos y a los que pueden ser vulnerables a coerción o influencia indebida". La propuesta actual (P19) agrega que "Todos los grupos vulnerables necesitan que se les considere protección específica".

\section{Investigación en población o comunidades vulnerables}

Párrafo 17: "La investigación médica en una población o comunidad con desventajas o vulnerable sólo se justifica si la investigación responde a las necesidades y prioridades de salud de esta población o comunidad y si existen posibilidades razonables de que la población o comunidad, sobre la que la investigación se realiza, podrá beneficiarse de sus resultados". Este párrafo también ha sido fuente de extensa controversia, especialmente en la medida que aumenta la investigación en países en vías de desarrollo, sin que necesariamente sea dirigida a resolver un problema local. La DdH actual incluye a las comunidades, dándole importancia a su expresión de necesidad y prioridad. La forma en que puede beneficiarse el país huésped puede no ser directamente relacionada con beneficios médicos para los individuos que participaron en el estudio, sino que puede también estar abierta a posibles mejoras en las condiciones de vida de la población o de ciertos grupos, como ha ocurrido en los estudios de vacunas contra la hepatitis E realizados por la Fuerza Aérea Estadounidense en miembros de las Fuerzas Armadas de Nepal, quienes obtuvieron como compensación apoyo militar y financiero ${ }^{15}$. Esto plantea problemas éticos, puesto que los beneficios no necesariamente son percibidos por quienes soportaron las cargas del estudio. Por otra parte, la frase "podrá beneficiarse de sus resultados" está en condicional, por lo que puede interpretarse en el sentido que en el futuro cualquier intervención podría beneficiar a los individuos de cualquier país, dejando de ser esta una restricción relevante. Por último, cabe señalar que no se especifica por qué sólo la investigación en población vulnerable debe estar justificada en base a las necesidades de la comunidad, puesto que toda investigación debiese justificarse según las necesidades locales ${ }^{11}$. En la DdH que está ahora en discusión (P20), se mantiene este párrafo y se agrega como condición que "la investigación no puede ser llevada a cabo en una población no vulnerable" " "Debería considerarse la posibilidad de asegurar que la comunidad reciba un nivel justo de beneficios adicionales". A nuestro juicio, este último párrafo no debiese estar en condicional, sino que debe asegurarse que la comunidad reciba un nivel justo de beneficios adicionales. 


\section{Investigación en personas con discapacidad física o mental}

Párrafo 27 (P28 de la DdH en revisión): "Para un sujeto potencial de investigación que sea incompetente, el médico debe obtener el consentimiento informado de un representante legal autorizado. Estos individuos no se deben incluir en una investigación que no tenga la probabilidad de beneficio para ellos, a menos que se pretenda promover la salud de la población representada por el sujeto potencial, la investigación no puede en cambio ser realizada con personas competentes y la investigación conlleva sólo riesgos minimos y problemas mínimos". La DdH 2008 agregó la frase respecto de riesgos mínimos y problemas mínimos, que no estaba contemplada en versiones anteriores, lo que significa que no debe exponerse al individuo a riesgos que no sean los mínimos, aun cuando los resultados de la investigación pudiesen beneficiar a futuros pacientes. A su vez, el Párrafo 28 (P29) especifica que "Si un potencial individuo que participa en la investigación es considerado incompetente pero es capaz de dar su asentimiento a participar o no en la investigación, el médico debe pedir dicho asentimiento, además del consentimiento del representante legal. El desacuerdo del potencial individuo debe ser respetado". El Párrafo 29 (P30) señala en uno de sus puntos que "la investigación en individuos que no son capaces física o mentalmente de otorgar consentimiento, por ejemplo los pacientes inconscientes, se puede realizar sólo si la condición física/mental que impide otorgar el consentimiento informado es una característica necesaria de la población investigada".

En consecuencia, la DdH contempla explícitamente la posibilidad de realizar investigación en sujetos que no están en condiciones de otorgar un CI válido, en la medida que la investigación se relacione directamente con la enfermedad de base. La legislación chilena, en cambio, es más restrictiva, puesto que la Ley 20.584 señala en su Artículo 28 que "Ninguna persona con discapacidad psíquica o intelectual que no pueda expresar su voluntad podrá participar en una investigación científica. En los casos en que se realice investigación científica con participación de personas con discapacidad psíquica o intelectual que tengan la capacidad de manifestar su voluntad y que hayan dado consentimiento informado, ... será necesaria la autorización de la Autoridad Sanitaria competente, además de la manifestación de voluntad expresa de participar tanto de parte del paciente como de su representante legal". Por lo tanto, esta legislación excluye la posibilidad de realizar investigación en personas con discapacidad psíquica o intelectual que no puedan expresar su voluntad ${ }^{9}$, lo que a nuestro juicio limita que se pueda contribuir en Chile a la investigación en métodos diagnósticos o terapéuticos para estas poblaciones. Así, la legislación chilena aparece enfatizando la protección del sujeto de investigación contra potenciales daños o riesgos, mientras que el concepto moderno de justicia en investigación señala la necesidad de asegurar acceso a los potenciales beneficios que la investigación tiene que ofrecer. Una evidencia clara de este cambio de paradigma lo ejemplifica la posibilidad de obviar el requerimiento de CI en situaciones de emergencia, dispuesto en las regulaciones federales de Estados Unidos de Norteamérica de $1996^{16}$.

\section{Consideraciones respecto de medidas al término del estudio}

El final del Párrafo 14 especifica que: "El protocolo debe describir los arreglos para el acceso después del ensayo a intervenciones identificadas como beneficiosas en el estudio o el acceso a otra atención o beneficios apropiados". Este punto hace exigible que al término del protocolo haya acceso a procedimientos o tratamientos que hayan sido considerados como beneficiosos. Estas medidas deben quedar descritas en el protocolo y en el CI, y deberán ser evaluadas por los respectivos comités de ética. La compensación al término fue motivo de debate también en la versión anterior de la DdeH, originando una clarificación al antiguo Párrafo 30. En todo caso, en qué consiste exactamente el acceso al término del protocolo es un aspecto que cada comité de ética deberá ponderar. Es necesario tener en cuenta que cargas excesivas a los patrocinadores pueden ser contraproducentes, encareciendo los costos de desarrollo de nuevos productos. Es probable que el comité de ética considere como aspecto importante si existe o no tratamiento alternativo, y se vea más inclinado a solicitar tratamiento de por vida para aquellas condiciones en las cuales no había terapia conocida como efectiva o si el sistema de salud local no puede proveerla. En esta línea, el Párrafo 33 señala: "Al final de la investigación, todas las personas que participan en el estudio tienen derecho a ser informadas sobre sus resultados y compartir 
cualquier beneficio, como por ejemplo, acceso a intervenciones identificadas como beneficiosas en el estudio o a otra atención apropiada o beneficios". Este párrafo también ha recibido críticas, puesto que se ha considerado que no va en el mejor interés del sujeto de investigación reemplazar el acceso a intervenciones identificadas como beneficiosas por "otra atención apropiada o beneficios". Esta forma de redacción deja abierta la posibilidad que las compensaciones correspondan a otro tipo de beneficios, lo que puede afectar los derechos de las personas que participaron como voluntarios?.

La nueva propuesta (P34) clarifica estas dudas que plantea la DdH 2008, y fortalece el tema de las medidas al término del protocolo: "Antes de un ensayo clínico, los patrocinadores, investigadores $y$ gobiernos de los países huésped deben establecer disposiciones para el acceso al término del estudio para todos los participantes que todavía necesitan una intervención identificada como beneficiosa en el estudio. Esta información debe ser revelada a los participantes durante el proceso de consentimiento informado. Todos los participantes del estudio deben ser informados del resultado del mismo". Nos parece que este cambio protege en forma adecuada a los participantes que requieran al término del estudio de algún tipo de intervención considerada como beneficiosa. A nuestro juicio, los comités de ética tienen la responsabilidad de velar que estas medidas queden debidamente estipuladas en los CI y que se cuenten con procedimientos adecuados para implementarlas.

\section{Conclusiones}

Para algunos, la DdH 2008 limita la investigación biomédica al aumentar las exigencias respecto del uso de placebo. Para otros, si bien existen avances, hay demasiados vacíos de interpretación, sobre todo respecto de las exigencias al término del estudio. La AMM está consciente de estos problemas y está realizando reuniones de expertos para revisar aspectos relacionados con investigación en población vulnerable, acuerdos al momento de finalizar el protocolo, y el tema de los bancos biológicos, entre otros ${ }^{17}$.

En nuestra opinión, la versión 2008 tiene imprecisiones en temas fundamentales, sobre todo en aquellos relacionados con las medidas al término del protocolo, que ameritan una nueva revisión.
Estimamos que la propuesta que está en revisión constituye un avance, puesto que provee un mayor grado de protección a los participantes de la investigación, manteniendo una redacción general acorde con lo que se espera de una Declaración a ser tomada en consideración a nivel mundial.

En resumen: Concordamos en que las restricciones al uso de placebo se mantengan; creemos que toda investigación debe justificarse según las necesidades locales; promovemos que se pueda hacer investigación en sujetos que no tienen capacidad para consentir, siempre y cuando la investigación vaya dirigida a promover la salud de la población representada por el sujeto potencial y, por lo tanto, no pueda ser realizada en sujetos sin la condición; y por último, creemos que la propuesta nueva de la $\mathrm{DdH}$ fortalece el tema de las medidas al término del protocolo, estableciendo en los protocolos las formas en que los participantes que todavía necesitan una intervención identificada como beneficiosa en el estudio tendrán acceso a ésta.

\section{Referencias}

1. Declaración de Helsinki-Principios éticos para las investigaciones médicas en seres humanos. Promulgada por la Asociación Médica Mundial en Seúl, Corea, octubre de 2008. Disponible en http://www.wma.net/ es/30publications/10policies/b3/index.html [consultado el 18 de noviembre de 2009].

2. Normile D. Ethics. Clinical trials guidelines at odds with U.S. policy. Science 2008; 322 (5901): 516.

3. Puri KS, Suresh KR, Gogtay NJ, Thatte UM. Declaration of Helsinki, 2008: implications for stakeholders in research. J Postgrad Med 2009; 55 (2): 131-4.

4. Williams JR. The Declaration of Helsinki and public health. Bull World Health Organ 2008; 86 (8): 650-2.

5. Goodyear MD, Eckenwiler LA, Ells C. Fresh thinking about the Declaration of Helsinki. BMJ 2008; 337: a2128.

6. Eckenwiler LA, Ells C, Feinholz D, Schonfeld T. Hopes for Helsinki: reconsidering "vulnerability". J Med Ethics 2008; 34 (10): 765-6.

7. Declaración de Buenos Aires sobre investigaciones médicas. Promulgada por la XI Asamblea Anual de la Confederación Médica Latinoamericana y del Caribe (Confemel). Buenos Aires, Argentina 2008. Disponible en http://www.confemel.com/asambleas/xiasam_bsas08_4. htm [consultada el 20 de diciembre de 2009]. 
8. Declaration of Helsinki. Ethical Principles for Medical Research Involving Human Subjects. World Medical Association Annotated draft for public consultation. 2013. Disponible en http://www.wma.net/en/20activi ties/10ethics/10helsinki/15publicconsult/index.html. [consultada el 19 de abril de 2013].

9. Ley Número 20.584. Regula los derechos y deberes que tienen las personas en relación con acciones vinculadas a su atención en salud. Promulgada por el Ministerio de Salud, Chile 2012. Disponible en http://www.leychile. $\mathrm{cl} / \mathrm{N} ? \mathrm{i}=1039348 \& \mathrm{f}=2012-10-01 \& \mathrm{p}=$ [consultada el 22 de abril de 2013].

10. Carta de Córdoba sobre ética en investigaciones en seres humanos. Promulgada en el II Congreso de la Red Latinoamericana y del Caribe de Bioética -Redbioética/ Unesco, 14 de noviembre de 2008. Córdoba, Argentina. Disponible en http://www.femeba.org.ar/view_doc. php?tipo=fun_docs\&archivo $=0000000526$.pdf [consultada el 25 de octubre de 2012].

11. Macklin R. The Declaration of Helsinki: another revision. Indian J Med Ethics 2009; 6 (1): 2-4.
12. Carpenter WJ Jr, Appelbaum PS, Levine RJ. The Declaration of Helsinki and Clinical Trials: A Focus on Placebo-Controlled Trials in Schizophrenia. Am J Psychiatry 2003; 160 (2): 356-62.

13. Salas SP. Aspectos éticos de la investigación biomédica: Desafíos actuales de la investigación en países en vías de desarrollo. Ars Médica 2006; 13: 11-24.

14. Diccionario etimológico de Chile. Disponible en http:// etimologias.dechile.net [consultado el 22 de abril de 2013].

15. Andrews J. Research in the ranks: vulnerable subjects, coercible collaboration, and the hepatitis E vaccine trial in Nepal. Perspect Biol Med 2006; 49 (1): 35-51.

16. Mastroianni A, Kahn J. Swinging on the pendulum: Shifting views of justice in human subjects research. En Law and ethics in biomedical research: Regulation, conflict of interest, and liability. Editado por Trudo Lemmens and Duff R Waring. University of Toronto Press; Toronto, Canadá 2006 p. 47-60.

17. Macklin R. Revising the Declaration of Helsinki: a work in progress. Indian J Med Ethics 2012; 9 (4): 224-6. 